\title{
Using room acoustical parameters for evaluating the quality of urban squares for open-air rock concerts
}

\author{
P. Thomas*, T. Van Renterghem, D. Botteldooren \\ Ghent University, Department of Information Technology, Sint-Pietersnieuwstraat 41, \\ B-9000 Gent, Belgium
}

\begin{abstract}
Sound quality research of urban squares used for open-air (rock) concerts is very scarce. In contrast to the study of (classical) concert halls, little is known about useful design parameters. For the design of the amplification system, the sound engineer currently often takes into account the desired sound pressure level only.

In this study, the ability of existing room acoustical parameters to characterize urban squares acoustically is investigated. An independent parameter set is identified for specific use on such squares. Besides the distribution of the sound pressure level over the square, different impulse response related parameters such as the clarity, center time, reverberation time and bass ratio were considered. In addition, binaural measures were included to measure qualities related to human spatial hearing.

This study is based on a measurement campaign, performed at five squares in Belgium before and during life rock concerts. Special attention was paid to the signal processing methodology, given the significant amount of en-
\end{abstract}

\footnotetext{
${ }^{*}$ Corresponding author

Email address: pieter.thomas@intec.ugent.be (P. Thomas)
} 
vironmental noise often found at such squares during measurements. The variation of these parameters is investigated in relation to the square geometry, the amplification set-up and the presence of delay-lines. Parameters like $C_{80}, T_{30}, I A C C_{E 3 / L 3}$ and $\Delta L_{e q, A / C}$ were shown to be very useful when characterizing the sound field at urban squares.

Keywords: urban rock concert, concert hall parameters, sound field description, impulse response measurement

\section{Introduction}

Over the last few decades the number of open-air concerts has grown a lot. Because of their historical character and social function, public squares are frequently used for concerts, despite the fact that these squares were not designed to hold such concerts and their acoustic quality is mostly far from ideal.

The difference with concert halls is significant [1]. Most squares have a typically large irregular shape and are bordered with acoustically quite rigid surfaces, whose degree of scattering varies to a large extent. The shape and location of scatterers is usually not adapted to the location of sources and listeners during concerts. Also the absence of a roof results in a high energy loss. In practice, this means that only a limited amount of reflections reach the listener. The influence of the audience is also apparent. In contrast to a concert hall, the public on a square is standing or even seated on the ground with varying density along the square. This results in a fluctuating absorption coefficient $[2,3]$.

In order to cope with these shortcomings, sound engineers have to design an 
amplification system that optimizes the sound field of the square, since the geometrical and acoustical properties are fixed. This amplification system mostly consists of different speakers or line-arrays, each speaker trying to optimize the sound field in a part of the square. For this purpose, delay-lines are used. These are line-arrays placed along the square, reproducing a delayed version of the sound signal.

For years now, the preferred and only design parameter for these amplification systems has been the sound pressure level SPL $[4,5]$. However, this parameter only describes the loudness of the sound and proves to be insufficient in giving full information on how the listener experiences the sound field. For classical concert halls, many studies have identified different sets of statistically independent parameters that determine the acoustical quality. The Goettingen school found the inter-aural cross-correlation $I A C C$, reverberation time $T_{30}$ and clarity $C_{50}$ to be the dominating factors [6]. Cremer et al. determined the strength $G$, bass ratio $B R$ and $C_{80}$, related with loudness, timbre and clarity as a set of representative parameters [7]. In 1983, Ando proposed four objective orthogonal factors that are related to subjective preferences, which he combined into a rating scale for concert halls $[8,9]$. These four factors consist of three temporal factors; the initial time-delay gap ITDG (related to intimacy), $G$ (related to loudness), $T_{\text {sub }}$ (related to reverberance) and a spatial factor namely the inter-aural cross-correlation $I A C C$ (related to the difference between left- and right-ear signals). In addition to these factors, Beranek used two more statistically independent parameters; the surface diffusion index $S D I$ and bass ratio $B R[10,11]$. By studying the acoustics of 35 concert halls, Gade showed in 1991 that the early decay time 
$E D T, T_{30}$ and $C_{80}$ are highly correlated, but orthogonal to the lateral fraction $L F$ and $G$ [12]. In a more recent study of 2009, Cerda measured the acoustical parameters at many locations in different types of performance halls in order to investigate the correlation of different acoustical parameters [13]. By using factor analysis he obtained a set of three orthogonal factors; the reverberation-intelligibility-clarity factor, the spatiality-subjectivity factor and the strength factor, respectively represented by $T_{30}$, Beranek's binaural quality index $(B Q I[10])$ and $G$.

Until now, no such studies have been undertaken to identify a statistically independent set of parameters for square-acoustics. It is therefore useful to investigate the existing parameters as mentioned in the ISO3382 standard [14] and extract those which can deliver more information on the sound field. Parameters such as the clarity $C_{80}$ and center time $T_{s}$ give us insight in how the received acoustical energy is distributed over time. Other parameters such as the early decay time $E D T$ and reverberation time $T_{30}$ are useful when describing the sound energy decay on the square over somewhat longer time scales. These parameters are extended with binaural parameters related to a subjective experience of apparent source width $A S W$ and listener envelopment $L E V$ to include the spatial impression of the sound field. The initial time delay gap ITDG [15] is, in the particular settings of outdoor amplified rock concerts, strongly determined by the (distributed) loudspeaker system, rather than by the acoustics of the space. As such, it correlated very strongly with other parameters measuring the fraction of early energy such as $C_{80}$. Therefore, this parameter was not considered explicitly. Other parameters such as the speech transmission index STI, lateral fraction $L F$ 
and strength factor $G$ could be useful as well, but were not considered in this study. However, an alternative formulation of the strength factor $G$ is proposed by using a relative sound pressure level.

In this paper, a set of statistically independent parameters for specific use on urban squares during rock-festivals is identified by correlation analysis. Their dependence on the geometry of the square and the used amplification system is then investigated with special attention for the influence of delay-lines on the parameters. This will be accomplished by comparing results of an extensive measurement campaign at four urban squares during and before rock concerts. The influence of the delay-lines is investigated by measurements at an open festival site in order to eliminate the influence of the geometry. A study of the subjective attributes and listener preferences related to these objective parameters is outside the scope of this research.

The measurements of the impulse response and related parameters are based on the ISO3382-1 and ISO18233 standard $[14,16]$. Special attention is given to the choice of test signal and signal (post-)processing.

\section{Parameter definitions}

The parameters which will be investigated are mainly extracted from the ISO3382 standard [14] and already well-known in the world of (concert-hall) acoustics [10]. This section gives a short overview, highlighting some slight variations in their definition.

\subsection{Energy related parameters}

In the introduction the strength factor $G$ was mentioned. The parameter $G$ measures the ratio of the sound field generated by an omnidirectional 
loudspeaker on stage at different locations in the room to a reference value recorded in an anechoic chamber at $10 \mathrm{~m}$ distance. In outdoor rock concerts, the directional speaker set-up has to be considered as part of the acoustical source. Therefore, the strict definition of $G$ based on an omnidirectional loudspeaker on stage and a reference point at $10 \mathrm{~m}$ makes little sense. As an approximation, we define the relative sound pressure level $\Delta L_{e q, o c t}$, in octave bands, as the difference between the A- or C-weighted sound pressure level at a position of interest and the weighted level at a certain reference point, usually chosen near the sound engineer. $\Delta L_{\text {eq,oct }}$ has values for each octave band. To have a single-number value, $\Delta L_{e q, A / C}$ is defined as the difference between the frequency summed A- or C-weighted sound pressure level $L_{e q, A / C}$ at the measurement position and $L_{e q, A / C}$ at the reference point. It can be seen that $\Delta L_{e q, o c t}$ and $\Delta L_{e q, A / C}$ are determined by the combined effects of frequency dependent path loss, audience absorption and reflection and absorption at the square edges. These parameters also incorporate the influence of the speaker radiation patterns and delay-lines.

In the ISO3382 standard three other important parameters are listed that are related to how the sound energy is divided over the early and late part of the sound signal. These are the Deutlichkeit $D_{50}$, clarity $C_{80}$ and center time $T_{s}$.

The Deutlichkeit and clarity are defined by the ratio of early-to-late arriving energy. However, the Deutlichkeit will not be included in this work, as it applies more to speech conditions. $C_{80}$ is more suitable for music signals, as the early energy is defined as the sound energy arriving within the first 
$80 \mathrm{~ms}$. There is however some discussion about the time which separates the early reflections from the late ones. Literature review shows that this separation time has values between $50 \mathrm{~ms}$ and $200 \mathrm{~ms}$. In their work, Hidaka and Beranek chose the separation limit for the early sound field to be at $80 \mathrm{~ms}$, based on own research and findings from Barron and Marshall $[17,18,19]$. Bradley and Soulodre used the same separation time [20]. However, in later

research, Soulodre found the separation time to be frequency dependent and proposed a set of perceptually motivated integration limits, based on the temporal integration properties of the hearing system [21]. In a 2008 paper by Ryu, the correlation between $C_{x}$ is investigated for $10 \mathrm{~ms} \leq x \leq 200 \mathrm{~ms}$ [22]. It is found that $C_{80}$ is significantly correlated with $C_{x}$ for $60 \mathrm{~ms} \leq x$, and $80 \mathrm{~ms}$ is chosen as separation limit. Based on these findings and to make comparisons with measured data from concert halls possible, it is decided in this work to opt for the ISO3382 definition of $C_{80}$ [23].

This discussion can be circumvented when using center time $T_{s}$. $T_{s}$ can be interpreted as the point of gravity of the acoustical energy over time, so no division of the impulse response between early and late sound has to be made.

\subsection{Reverberation related parameters}

The reverberation time is most often used by acousticians to describe the energy decay of the sound field. It is defined as the amount of time it would take for the sound field to attenuate $60 \mathrm{~dB}$, given the slope of the level function. This level function is determined by Schroeder as the backwards integrated squared impulse response [24]:

$$
L(t)=10 \log _{10} \int_{t}^{\infty} h^{2}(x) d x+C_{t e} \quad[d B],
$$


where $h(x)$ is the measured impulse response and $C_{t e}$ accounts for the reference pressure and signal power. $C_{t e}$ will be omitted in further discussions. Since this level function can have several slope changes, alternative definitions of the reverberation time were postulated. In this study, the early decay time $E D T$ and $T_{30}$ are used.

In general, the sound field of a concert hall is conditioned in such a way that the level function is linearly descending in time, so the EDT and $T_{30}$ have more or less the same value [1]. On an open square this is hardly the case. On these squares, there are only reflections coming from the sidewalls. Because of the size of the squares, only the direct sound and discrete early reflections contain most of the energy, resulting in a kind of sagging decay. The measured EDT will prove to be more location dependent. $T_{30}$, however, has a more or less constant value over the square, since this parameter is determined by later parts of the reverberant sound field.

An important derived descriptor of the reverberation time is the bass ratio $(B R)$, which allows to investigate the frequency dependencies of the reverberation time:

$$
B R_{R T}=\frac{R T_{125 \mathrm{~Hz}}+R T_{250 \mathrm{~Hz}}}{R T_{500 \mathrm{~Hz}}+R T_{1000 \mathrm{~Hz}}} \quad[-]
$$

\subsection{Binaural related parameters}

With the studies of Barron and Marshall the importance of spatial impression was introduced; it describes how the listener experiences the spaciousness of the sound field [19]. They identified factors that relate to the spatial impression, composed of two components: the apparent source width and listener envelopment. Since then, the correlation of the subjective quantities $A S W$ and $L E V$ with variants of objective parameters such as $L F, I A C C$, 
$G$ and $L G$ has been investigated in many studies $[17,18,19,20,21,25,26,27]$.

\subsubsection{Objective measures for apparent source width $A S W$}

The apparent source width $A S W$ is a measure for the auditory width of the sound field at the square. Early reflections, arriving from the sides, tend to broaden the source, so the stage gains auditory width.

Objective parameters for $A S W$ include $L F_{E},\left[1-I A C C_{E}\right]$ and $G_{l o w}[25,26]$. In this study we will use $\left[1-I A C C_{E 3}\right]$ as representation for $A S W$. Here, the subscript $E 3$ means that $I A C C$ is calculated from the early sound field (first $80 \mathrm{~ms})$ at the three mid-frequency octave bands $(0.5,1,2 \mathrm{kHz})$. Beranek called this factor the binaural quality index $B Q I$ and it was found to be a better measure of $A S W$ than $L F_{E 4}$ [23].

\subsubsection{Objective measures for listener envelopment LEV}

The listener envelopment $L E V$ describes the directionality of the reverberant field and expresses how the listener feels emerged in the sound field. It is known that the listener prefers to be enveloped in the sound field [28,p.23]. Different measures for $L E V$ can be found. From Bradley and Soulodre it is known that $L E V$ is mostly influenced by the level and direction of arrival of late reflections, arriving $80 \mathrm{~ms}$ after the direct sound [20]. It was concluded that $L E V$ correlates well with $I A C C_{L}$ and $L F_{L}$, with respect to the influence of angular distribution of the late sound. To combine the influence of the level with angular distribution, the late lateral sound level $L G_{L 4}$ was defined. In later work, Soulodre found that it was better to give separate weightings to both the level component and spatial component [21]. Furthermore, he also introduced perceptually motivated integration limits for the time sepa- 
ration between early and late arriving sound, as discussed in section 2.1. A new measure $G S_{\text {perc }}$, based on a linear combination of both measures and using the separation times, was proposed [21].

In this research we will examine $\left[1-I A C C_{L 3}\right]$, with the subscript $L 3$ restricting $I A C C$ to the late sound (starting from $80 \mathrm{~ms}$ ) and the three mid-

frequency octave bands. A second measure, based on an adaptation from Beranek on Soulodre's work, will also be used. Beranek uses the following formula to calculate an objective value for $L E V$ :

$$
L E V_{\text {calc }}=0.5 G_{L 3}+10 \log _{10}\left(1-I A C C_{L 3}\right) \quad[d B]
$$

with $G_{L}=G-10 \log _{10}\left(1+\log _{10}^{-1} C_{80} / 10\right)$ [23]. However, since $G$ could not be measured in this study, $\Delta L_{e q, o c t}$, as defined in section 2.1, will be used instead.

\section{Measurement methodology}

For the measurement of the different quantities, numerous measurement techniques have been developed. However, since most of the described parameters are extracted from the impulse response (by using a HATS or omnidirectional microphone), it is worthwhile to optimize this impulse response measurement. Different methods have been described [29] and standardized [16]. However, for the choice of the test signal, deconvolution technique and post-processing, it should be kept in mind that these measurements will be conducted at large open squares, where the levels of background noise are often high and the transfer functions are not time invariant. 


\subsection{Test signal and deconvolution technique}

In order to measure the impulse response, a well-known test-sequence is emitted by the loudspeakers. The commonly used set of test signals can be divided into two groups. A first group uses noise sequences, which excite the full audio frequency range at the same time. White or pink noise sequences and Maximum Length Sequences could be used. However, this category seems unfit to use at an open square. Since the levels of background noise are expected to be high at a square, high signal levels should be produced. Furthermore, an ensemble average should be taken, so many impulse response measurements are required. The use of a MLS overcomes these problems, but this signal is very sensitive to time variations in the transfer function (wind, temperature changes, small movements...) and will not be used.

The second class of test signals are the frequency sweeps, linear or exponential. The main advantage is that a sweep excites only one frequency at the time, so the available power is not spread over the full frequency range compared to the noise test sequences. This results in an excellent signal-tonoise ratio (SNR). Prolongation of the sweep increases the SNR. Repetition of the sweep is however not advisable, since the corresponding frequency components of each repeated sweep are spread in time and undergo different changes due to the time varying character of the system. An exponential sweep of $12 \mathrm{~s}$ with frequencies ranging from $10 \mathrm{~Hz}$ to $16 \mathrm{kHz}$ is chosen. This signal duration corresponds to three times the estimated reverberation time. The exponential sweep is chosen over the linear sweep, so that each octave band is excited with an equal amount of energy. 
The extraction of the impulse response out of the recorded sweep (deconvolution) is done by spectral division of the recorded spectrum and the source spectrum, measured at the mixing console. However, since the source signal does not contain any frequency components between $16 \mathrm{kHz}$ and $22.05 \mathrm{kHz}$ (half of the sampling frequency), care should be taken to exclude this frequency range from the analysis.

\subsection{Measurement set-up}

In order to investigate the variation of the sound field along the square, it is essential that the described acoustical parameters are measured at different locations. The position of these locations, typically five or six, is based on the ISO 3382-standard [14]. Here it is stated that the measurement locations should be chosen randomly over the square, at a minimal distance of a quarter-of-wavelength from each other (min. $2 \mathrm{~m}$ ). Also a minimum distance of $7 \mathrm{~m}$ from the speakers will be maintained.

The measurements at each selected position are performed in two phases. First, the impulse response measurements and binaural measurements are done. Because it is impossible to play a test sequence like a sweep during a concert, these measurements took place before the start of the concert. At that moment the background noise was lower. For the determination of the impulse response, the exponential sweep is recorded with an omnidirectional 1/2-inch free-field microphone, type 4189 with a preamplifier type $2669 \mathrm{C}$, both of Brüel \& Kjær. This microphone is wind-shielded and its height is about $1.8 \mathrm{~m}$. The measurement of the binaural parameters is done at the same time, by recording the test-sequence with a head-and-torso simulator type $4128 \mathrm{C}$, in which two ear simulators type $4158 \mathrm{C}$ are mounted, also of 
Brüel \& Kjær. The signal of these three microphones is recorded simultaneously with the original sweep, as it was sent to the speaker system by the mixing console.

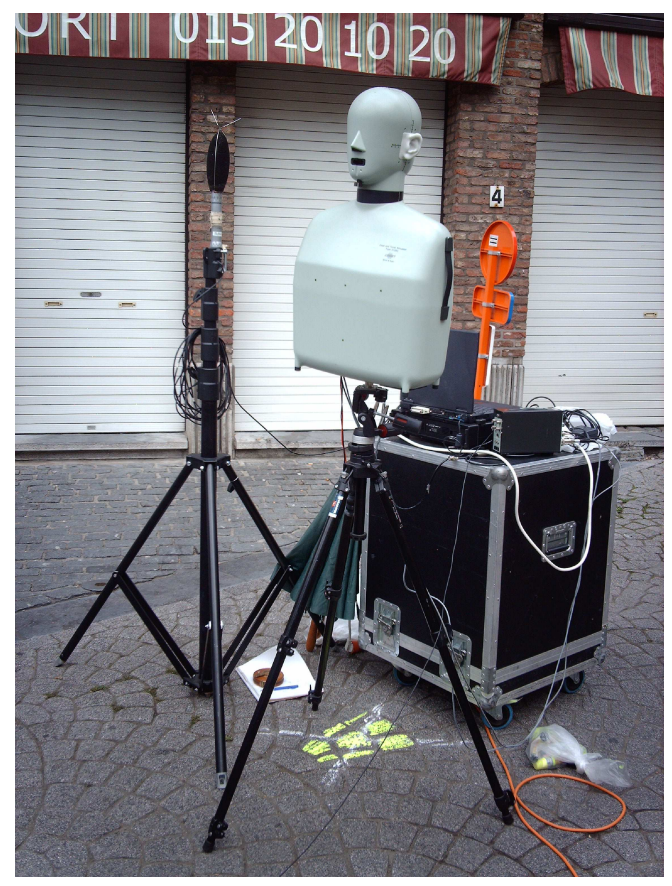

(a) Equipment used for the $I A C C$ and impulse response measurement.

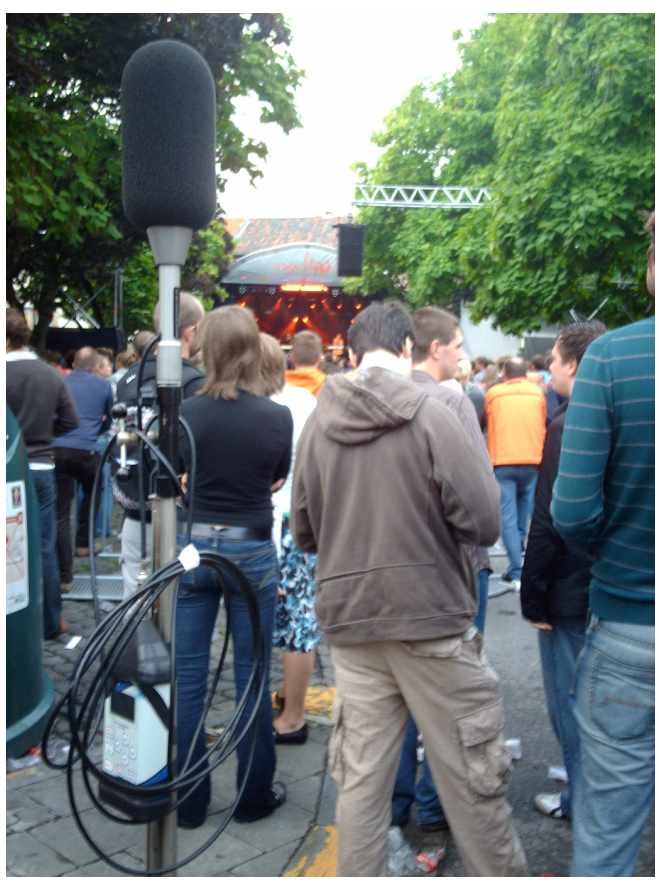

(b) Equipment used for the relative SPL measurement.

Figure 1: Measurement set-up before and during the concert.

In a second phase, the relative sound pressure level is measured. These measurements took place during the concert in order to include the influence of the audience. For this, a fixed reference SPL measurement is carried out simultaneously with a position dependent SPL measurement. For the reference measurement, a calibrated SVANTEK SVAN 959 soundanalyzer is used 
with a MK250 microphone and SV12L preamplifier. This measurement is carried out near the mixing console, where an open space of about $5 \mathrm{~m}^{2}$ is available. The position dependent SPL measurement is carried out with a calibrated SVANTEK SVAN 949 soundanalyzer with a similar microphone and preamplifier. At each position the SPL is logged during 10 min with an integration time of $1 \mathrm{~s}$. Out of each measurement only the useful parts, where the music is dominant, are selected. After $A$ - or $C$-weighting of the individual samples, the average energy per octave band is computed. An identical procedure is applied to the corresponding time intervals of the reference measurement. $\Delta L_{e q, o c t}$ is then calculated as the difference between the weighted sound levels per octave band at measurement and reference position, while $\Delta L_{e q, A / C}$ is calculated by subtracting $L_{e q, A / C}$ of both positions (see Section 2.1).

\subsection{Impulse response post-processing}

\subsubsection{Octave band filtering}

After extraction of the impulse response by spectral division, further signal processing is needed in order to extract reliable quantities. First, the impulse response has to be decomposed into its different octave bands, ranging from the $63 \mathrm{~Hz}$ to the $8 \mathrm{kHz}$-band. For this purpose a filterbank with $2 N^{t h}$ order Butterworth octave bandfilters is used, following the ANSI S1.11-2004 standard [30]. The use of $1 / 3^{\text {th }}$-octave bandfilters will be discarded. Because of their small bandwidth at lower frequencies $\left(11.5 \mathrm{~Hz}\right.$ for the $50 \mathrm{~Hz} 1 / 3^{t^{t h}}$ octave band), the corresponding filterresponse length will be long, since the bandwidth-time product is constant at a given filterorder. This can pose a problem because at low frequencies the measured impulse responses can 
be short, especially at large open squares with high losses. Since octave bandfilters have a larger bandwidth $(45 \mathrm{~Hz}$ at the $63 \mathrm{~Hz}$-octaveband) and thus a shorter filterresponse, they are more suitable. For the choice of the filterorder, this bandwidth-time product but also the filter characteristics have to be considered. A $2 N^{t h}$-order Butterworthfilter has a maximal flatness in the passband. In the stopband this filter attenuates according to $A_{t t}[d B] \approx 20 N \log _{10} \omega$, or equivalent, $6 N d B /$ octave. It is found that an $8^{\text {th }}$-order Butterworth octave filter $(N=4)$ has a bandwidth-time product that is small enough for the given octave bands and pass/stop characteristics that meet our requirements.

\subsubsection{Impulse response truncation}

The measured impulse responses will be inevitably contaminated with noise. When calculating the level function and other related parameters, this noise will pose a lower limit to the signal decay. Therefore, the impulse response is processed to select only the useful part. First, the starting point, $t_{0}$, of each band-response is determined. This is the time when the level function of the band-filtered impulse response starts to decay. This starting point can differ for each octave band, since not all frequency bands are reproduced by the same loudspeakers. The endpoint, $t_{1}$, can be found by using a truncation method described by Lundeby [31]. This algorithm is based on an estimation of the crosspoint of the decaying slope and a constant noise level of the time-averaged band-response. Originally, this procedure was designed to operate automatically. In some cases however, the estimation will be adapted manually, since peaks in the background noise can disturb the automatic procedure. 
For the calculation of the clarity and center time, the upper limit of the integral will be restricted by the finite length of the truncated band-response. Correction coefficients are introduced to account for this restriction [31]. These are based on the assumption that a regression line can be fitted to the exponential decay of the late energy. Knowing the slope $A$ and the offset $B$ at $t=0$ of the energy decay $h^{2}(t)=E(t)=B e^{A t}$, the compensation coefficients are calculated in eq. 4 , with $t_{1}$ the truncation point,

$$
\begin{gathered}
E_{\text {comp }}=B \int_{t_{1}}^{\infty} e^{A t} d t=-\frac{B}{A} e^{A t_{1}}, \\
T_{\text {comp }}=B \int_{t_{1}}^{\infty} t \cdot e^{A t} d t=-\frac{B\left(A t_{1}-1\right)}{A^{2}} e^{A t_{1}} .
\end{gathered}
$$

Using Schroeder's integrated impulse method [24], the level function can now be calculated from this restricted band-response. However, before the integration of $h^{2}(t)$, the mean-square value of the noise is subtracted from the squared restricted band-response to cope with faulty estimations of the truncation point [32]. For the binaural measurements no such corrections are applied, and only the truncated ear responses will be used.

\section{Case-studies}

\subsection{Description of the squares}

In this paper the acoustical quality of four different squares in Belgium, Europe, is measured. All four squares are situated in the city-center and are surrounded by different types of façades. Two squares are located in Leuven, where measurements were performed during 'Marktrock 2008' at the Oude Markt and the Vismarkt. At both squares, the amplification system was very 
elaborated. Besides speaker-arrays and subwoofers near the stage, different delay-lines were placed along the square. The Oude Markt is pictured in fig. 2(a). This elongated square is bordered by high $19^{\text {th }}$-century façades, and has dimensions of $160 m \times 85 \mathrm{~m}$. The Vismarkt (fig. 2(b)) is a smaller square with dimensions $85 m \times 40 m$ and big trees near the edges.

Two other squares were chosen in Mechelen during 'Maanrock 2008', namely the Grote Markt and the Vismarkt. No distributed speaker set-ups were used. All speakers, including subwoofers, are located beside or under the stage. The Grote Markt (fig. 2(d)) is the largest square, having dimensions of $100 \mathrm{~m} \times 85 \mathrm{~m}$. It is bordered by two storey buildings near the side while the backside of the square is partially open. The Vismarkt is the smallest investigated square (fig. 2(c)). The dimensions are $60 \mathrm{~m} \times 25 \mathrm{~m}$. At one side it is completely open due to its location near the river Dijle.

Measurements at a fifth square were used to illustrate the influence of the delay-lines on the acoustical parameters. This was possible since this square was very open with almost no reflecting boundaries. The square is situated near the city of Lokeren, where an artificial festival site was created for the 'Lokerse Feesten' (fig. 2(e)). The site was approximately $90 \mathrm{~m} \times 70 \mathrm{~m}$.

For easy notation the names of the squares will be abbreviated in the remainder of this paper: LO for Oude Markt (Leuven), LV for Vismarkt (Leuven), MG for Grote Markt (Mechelen) and MV for Vismarkt (Mechelen). 

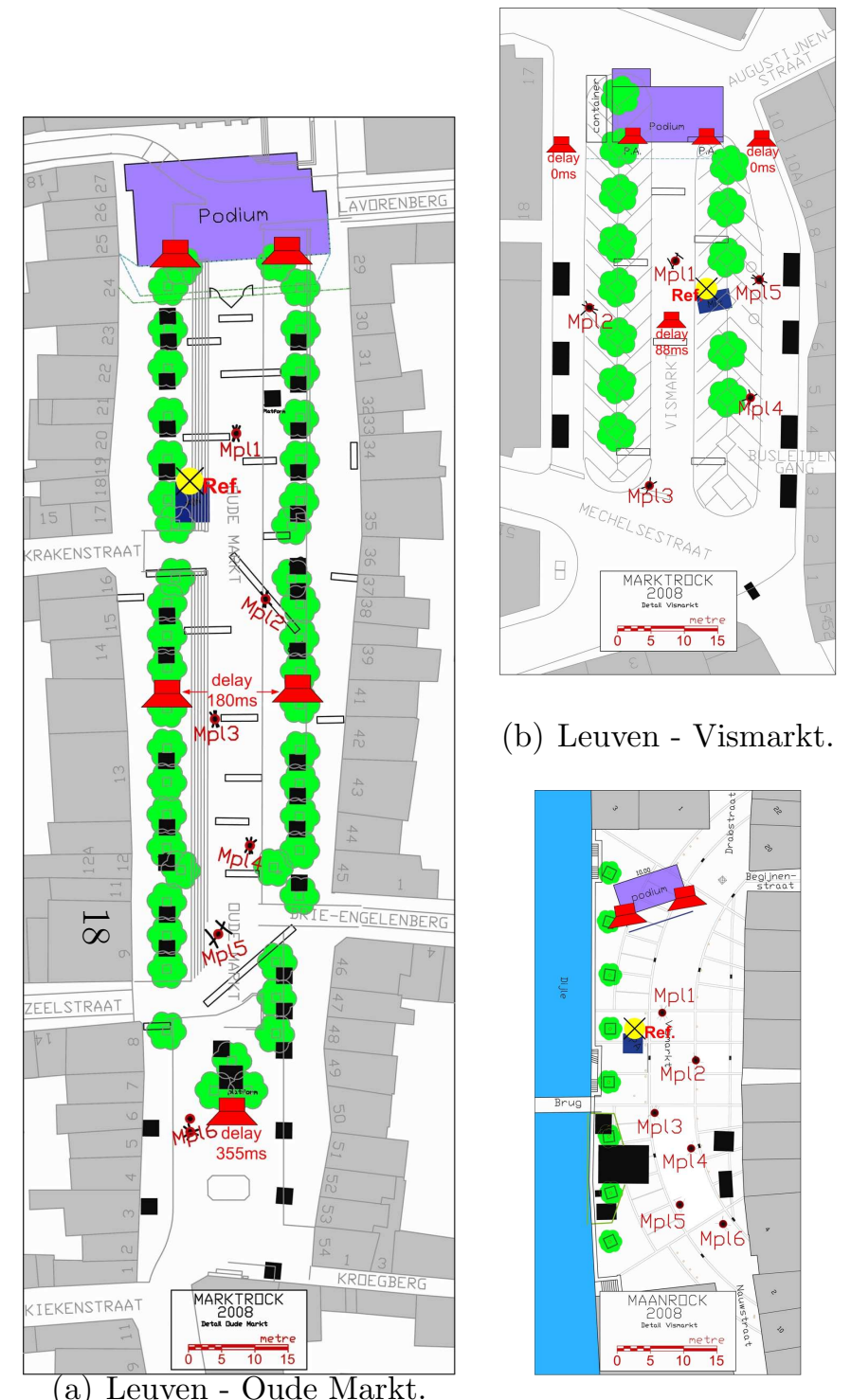

(b) Leuven - Vismarkt.

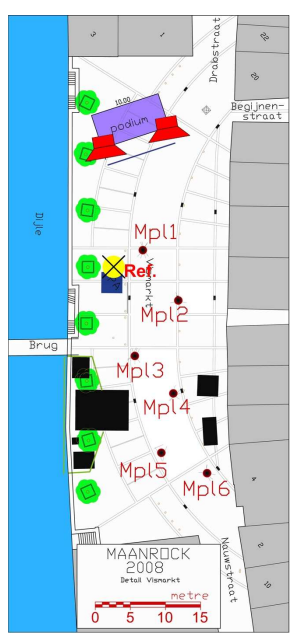

(c) Mechelen
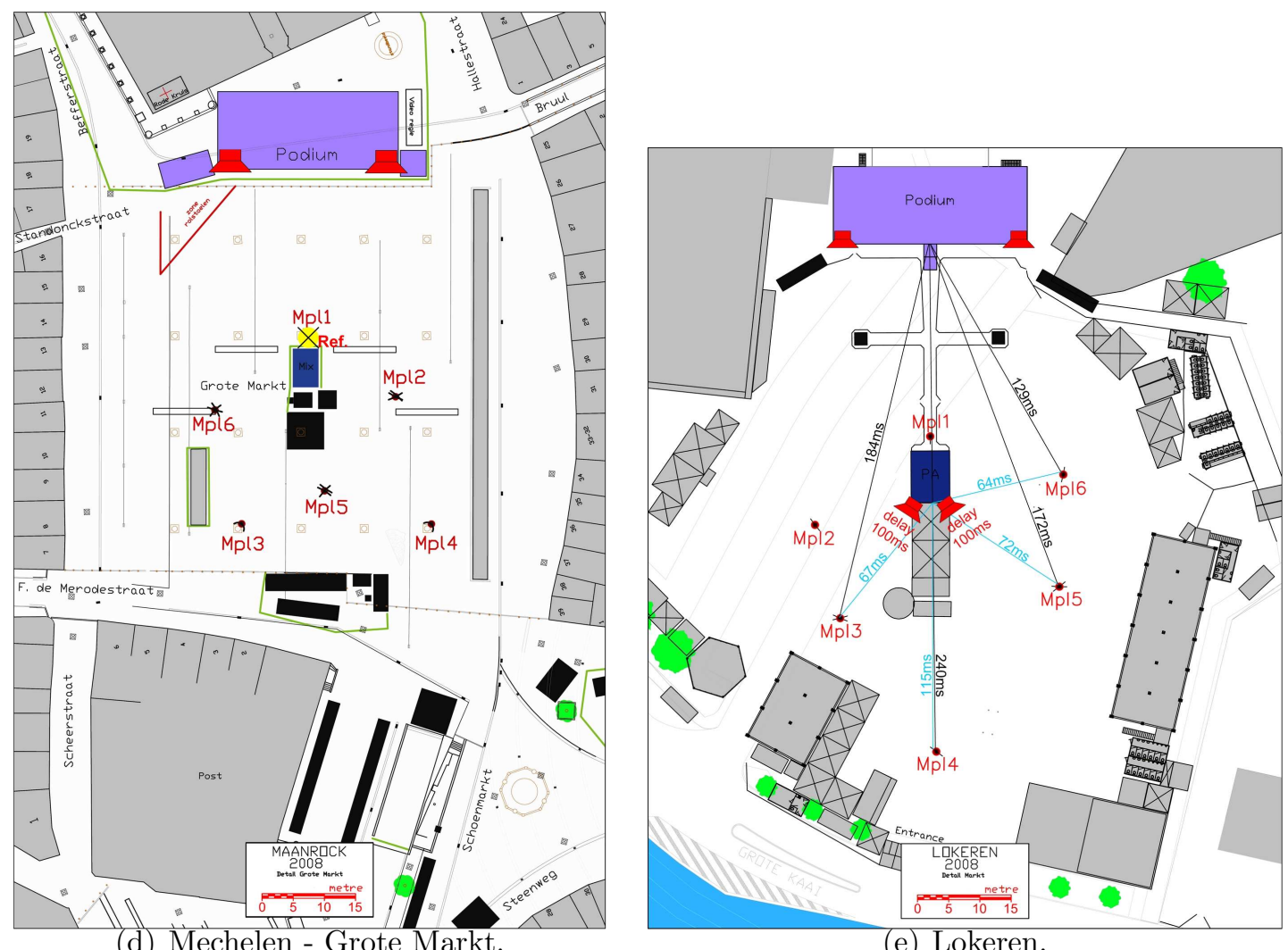

Vismarkt.

Figure 2: Five squares under test: two squares in Leuven during 'Marktrock 2008' (2(a) and 2(b)), two squares in Mechelen during 'Maanrock 2008' (2(d) and 2(c)) and one square in Lokeren during the 'Lokerse Feesten'. In red the measurement positions and speakers with delay-time are indicated. For the 'Lokerse Feesten' (2(e)), the estimated path delay is also given. The yellow dot with black cross indicates the reference position for the relative SPL measurement. The pink rectangular gives the stage's location. 


\subsection{Correlation analysis}

Before characterizing the sound field at the different squares, it is useful to define an independent set of parameters. Therefore, we perform an overall correlation analysis of the measured quantities at LO, LV, MG and MV. The parameters measured at the fifth open square will not be included, as this square is not representative for an enclosed urban square and is mainly investigated to characterize the influence of the delay-lines.

As already mentioned, the included parameters are the early response parameters $C_{80}$ and $T_{s}$, reverberation related parameters $E D T, T_{30}$ and $B R$, binaural parameters $\left[1-I A C C_{E 3}\right],\left[1-I A C C_{L 3}\right]$ and $L E V_{c a l c}$, and parameters related to the relative sound pressure level $\Delta L_{e q, o c t}, \Delta L_{e q, A}$ and $\Delta L_{e q, C}$. However, as $C_{80}, T_{s}, E D T, T_{30}$ and $\Delta L_{e q, o c t}$ are determined for each octave band, we will reduce the data by only correlating their single number averages, calculated from the mid frequency bands $(500 \mathrm{~Hz}-1 \mathrm{kHz})$, based on the ISO3382 standard [14]. 


\begin{tabular}{|c|c|c|c|c|c|c|c|c|c|c|c|}
\hline & $C_{80, a v g}$ & $T_{s, a v g}$ & $E D T_{a v g}$ & $T_{30, a v g}$ & $B R_{E D T}$ & {$\left[1-I A C C_{E 3}\right]$} & {$\left[1-I A C C_{L 3}\right]$} & $L E V_{\text {calc }}$ & $\Delta L_{e q, a v g}$ & $\Delta L_{e q, A}$ & $\Delta L_{e q, C}$ \\
\hline$C_{80, a v g}$ & - & & & & & & & & & & \\
\hline$T_{s, a v g}$ & $-0.92^{a}$ & - & & & & & & & & & \\
\hline$E D T_{a v g}$ & $-0.84^{a}$ & $0.81^{a}$ & - & & & & & & & & \\
\hline$T_{30, a v g}$ & $-0.49^{b}$ & $0.49^{b}$ & $0.68^{a}$ & - & & & & & & & \\
\hline$B R_{E D T}$ & -0.06 & 0.04 & -0.02 & -0.30 & - & & & & & & \\
\hline$\left[1-I A C C_{E 3}\right]$ & 0.16 & -0.01 & -0.31 & -0.41 & 0.18 & - & & & & & \\
\hline$\left[1-I A_{-} C C_{L 3}\right]$ & $0.48^{b}$ & -0.34 & -0.40 & -0.30 & 0.20 & 0.38 & - & & & & \\
\hline$L E V_{\text {calc }}$ & $-0.54^{b}$ & $0.59^{a}$ & $0.54^{a}$ & $0.59^{a}$ & 0.32 & -0.15 & -0.05 & - & & & \\
\hline$\Delta L_{e q, a v g}$ & 0.25 & -0.09 & -0.10 & 0.22 & 0.30 & -0.09 & 0.19 & $0.65^{a}$ & - & & \\
\hline$\Delta L_{e q, A}$ & $0.44^{b}$ & -0.28 & -0.32 & 0.00 & 0.26 & -0.04 & 0.28 & $0.46^{b}$ & $0.94^{a}$ & - & \\
\hline$\Delta L_{e q, C}$ & $0.55^{a}$ & $-0.48^{b}$ & $-0.73^{a}$ & $-0.73^{a}$ & 0.31 & 0.37 & 0.21 & -0.25 & 0.23 & $0.49^{b}$ & - \\
\hline
\end{tabular}

Table 1: Correlation coefficients $r$ between the different parameters, as calculated from measured values from measurement positions at LO, LV, MG and MV. Correlations where $|r| \geq 0.6$ are indicated in bold. 
In Table 1, it can be seen that $C_{80, a v g}, T_{s, a v g}$ and $E D T_{a v g}$ are highly correlated $(p<0.01)$. It is concluded that these parameters have similar fluctuations over the square, related to the delay-lines and geometry. $T_{30, \text { avg }}$, on the other hand, is correlated with $E D T_{a v g}$, but does not correlate with $C_{80, a v g}$ and $T_{s, a v g} . B R$ is uncorrelated with the considered parameters. Furthermore, apart from a correlation between $L E V_{\text {calc }}$ and $\Delta L_{e q, a v g}$ which are correlated by construction, the different binaural parameters are uncorrelated, not only with each other but also with the other objective parameters. For the parameters related to $\Delta L_{e q, o c t}$, only $\Delta L_{e q, C}$ is correlated with the reverberation related parameters and $\Delta L_{e q, A}$ is highly correlated with $\Delta L_{e q, a v g}$. It should be noted however, that there is little correlation between $\Delta L_{e q, A}$ and $\Delta L_{e q, C}$. From this it is concluded that both measures will have a different distribution over the square. Thus, including the lower frequencies by $C$-weighting, alters the behavior of the parameter significantly.

From the earlier defined parameters, we can now extract a statistically independent set of parameters, by using $C_{80}, T_{30}, B R,\left[1-I A C C_{E 3 / L 3}\right], L E V_{\text {calc }}$ and $\Delta L_{e q, A / C}$. This is more or less in accordance with the literature, except for $T_{30}$, which is, in performance halls, much more correlated with $C_{80}$ and $T_{s}[12,13]$.

\subsection{Influence of the delay-lines at the Lokerse Feesten}

To investigate the influence of the delay-lines apart from the influence of the square geometry, measurements were performed at an open festival site, with little to no reflecting boundaries. The speaker set-up consisted of two speaker-arrays at each side of the stage and a group of subwoofers beneath the stage. Two delay-lines were placed at the center of the site, 
which reproduced the sound with a delay of approximately $100 \mathrm{~ms}$. All four line-arrays reproduced the mid and high frequency octave bands but did not contribute to the lowest two octave bands $(63 \mathrm{~Hz}$ and $125 \mathrm{~Hz}$ ). Impulse response measurements were done at six locations along the square. At four of these positions, additional measurements were performed. First, all speakers were turned on, including the delay-lines at the center, with the delay set at the actual value. During the second measurement, only the speakers near the stage were used.
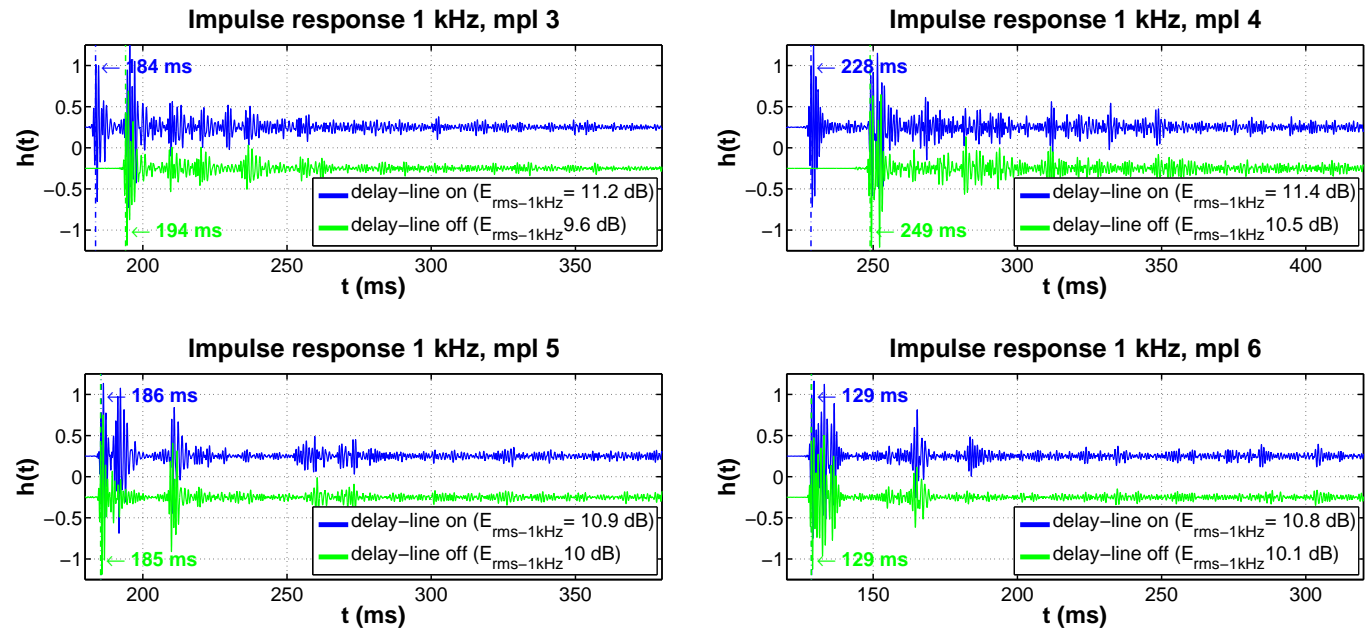

Figure 3: Impulse responses, with (blue) and without (green) delay-lines, at four measurement positions at the 'Lokerse Feesten' (fig. 2(e)). For both impulse responses the time of arrival of the first sound is indicated, together with the $E_{r m s}$-value of the $1 \mathrm{kHz}$ octave band.

Results of the impulse response measurements are depicted in fig. 3, with the actual time delay highlighted in the figure. For comparison, the estimated path travel-time, measured from the center of the stage, is given in 
fig. 2(e). Comparing both impulse responses shows that the time difference between the arrival of the sound of the main speakers and the delay-lines strongly depends on the position. At the $3^{\text {th }}$ and $4^{\text {th }}$ position, the sound of the delay-lines arrives earlier (10 ms @ MPL3 and $20 \mathrm{~ms} @$ MPL4). At the $6^{\text {th }}$ position, the first sound to arrive is coming directly from the stage. $50 \mathrm{~ms}$ later a delayed peak in the impulse response is seen, clearly weakened because of the off-axis position from the directive delay-lines. At the $5^{\text {th }}$ position, the arrival of the sound from both the stage and delay-lines is simultaneously.

This observation is due to have an effect on the acoustical parameters. Fig. 4 shows the effect of the delay-lines on $C_{80}$ as a function of frequency at the four selected locations on the square. At the lowest octave bands little or no effect is seen because these frequencies are produced at the same place, namely by the subwoofers beneath the stage and not by the delaylines. At higher frequencies, the differences when using delay-lines become clear. At MPL3 and MPL5 the clarity increases slightly. These are the two positions close to the line-arrays which have a minimal mismatch in time. At the other positions with a bigger mismatch, MPL4 and MPL6, the clarity becomes lower than in the absence of delay-lines. Analogous conclusions can be drawn from the extraction of the center time and early decay time (not shown).

Concerning $T_{30}$, differing conclusions are drawn. As is clear from previous research [33], $T_{30}$ changes only little over space. Fig. 5(a) shows that this is valid as well for a festival site, since the deviation from the median value is 

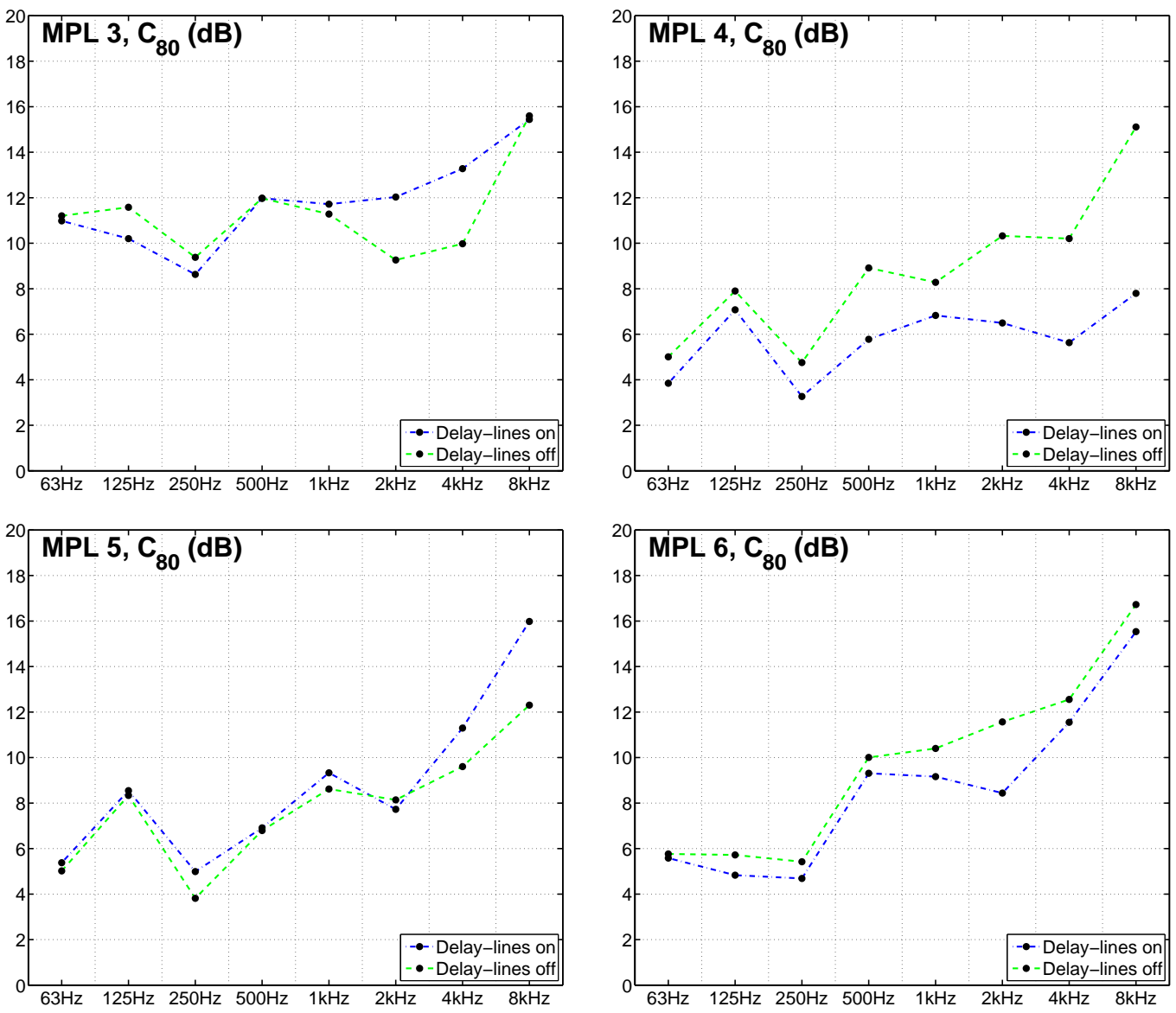

Figure 4: $C_{80}$ for each octave band for different measurement positions at the 'Lokerse Feesten' (fig. 2(e)), with (blue) and without (green) delay-lines.

relatively small. When delay-lines are used, only a limited decrease of $T_{30}$ in the mid frequency bands is observed.

The binaural quality index $B Q I$ or $\left[1-I A C C_{E 3}\right]$ is more influenced by the delay-lines. It is shifted to higher values because of the broadening effect of the delay-lines, directed to the sides of the square. For $\left[1-I A C C_{L 3}\right]$, no change is noticed since this measure is completely determined by the late reverberant sound field, which is not influenced by the delay-lines. This is 


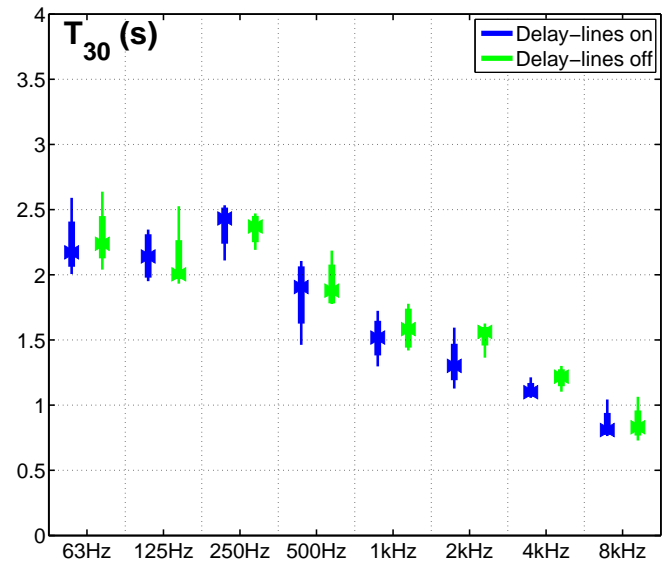

(a) $T_{30}(s)$

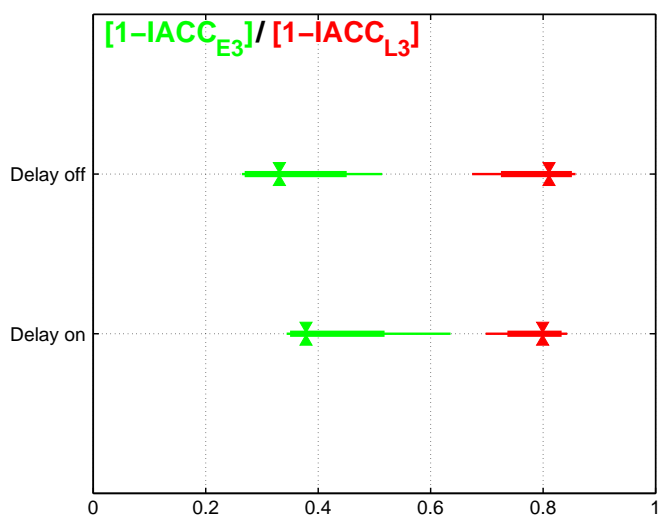

(b) $\left[1-I A C C_{E 3 / L 3}\right](-)$

Figure 5: $T_{30}$ and $\left[1-I A C C_{E 3 / L 3}\right]$ for MPL3-6 at the 'Lokerse Feesten' (fig. 2(e)) plotted in a boxplot in relation to the variation of the parameter over the square. The central mark is the median, the edges of the box are the $25^{\text {th }}$ and $75^{\text {th }}$ percentiles and the whiskers extend to the most extreme data points.

proved when looking at the impulse responses and $T_{30}$.

For $L E V_{\text {calc }}$ and $\Delta L_{e q, A / C}$ no investigations were made at this square.

This example shows that the introduction of delay-lines can improve the sound quality at the square. However, it should be accounted for that at more distant positions, the quality can worsen as the difference in arrival time grows. In between the main line-arrays and the delay-lines, the disturbing effects will be perceived less, because of the frontal directivity of the speakers, and this in spite of the larger time mismatch. 


\subsection{Comparison of 4 urban squares}

With the knowledge of the influence of the delay-lines at the open site, it is now possible to describe the sound field at urban squares, (partially) surrounded by façades. The chosen squares differ by geometry and amplification system (with or without delay-lines).

The following comparison is based on the statistically independent acoustical parameters measured at each square. Some of these acoustical parameters are function of the measurement position and octave band. To have a global view, the results will be grouped in a boxplot for an easy inter-square comparison. In this boxplot, the central mark indicates the median, the edges

of the box are the $25^{\text {th }}$ and $75^{t h}$ percentiles and the whiskers extend to the most extreme data points.

\subsubsection{Description by clarity $C_{80}$}

The first analysis is based on the clarity factor $C_{80}$ (fig. 6(a)) and, related with this factor, EDT (fig. 6(b)). In the discussion below, a distinction is made between the low octave bands $(63-250 \mathrm{~Hz})$, mid octave bands $(0.5-2 k H z)$ and high octave bands $(4-8 k H z)$.

For the low octave bands, large deviations of the measured quantities are seen at each square. This is a consequence of the centralized subwoofer system beneath the stage: the energy confined to the early sound decreases with the distance to the stage, while the reverberant energy increases due to the more frequent late reflections. In a later section, special attention is given to $\mathrm{MG}$, where the parameters at the $63 \mathrm{~Hz}$-band vary the most.

At mid and high octave bands the range in measured values becomes 


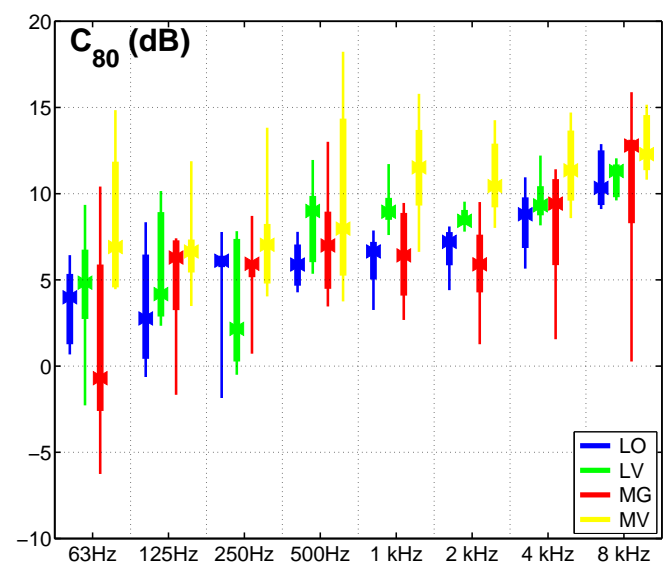

(a) $C_{80}(d B)$

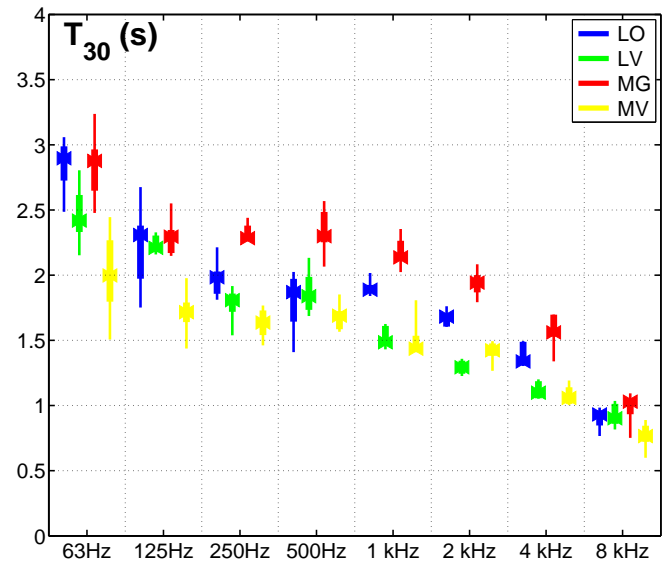

(c) $T_{30}(s)$

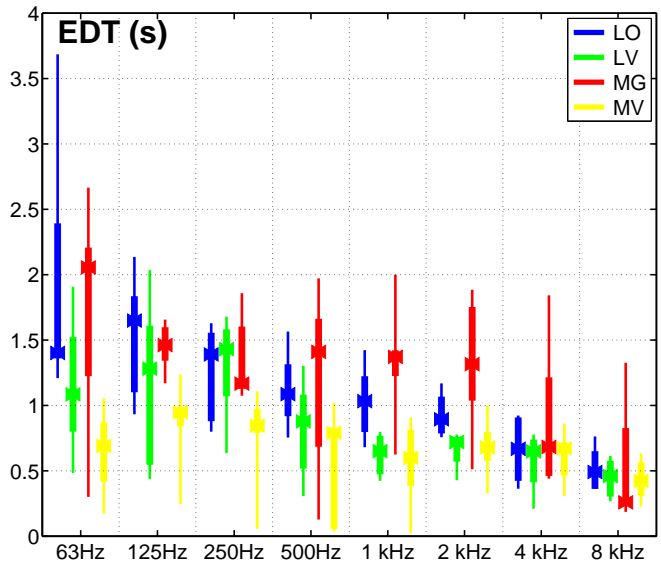

(b) $E D T(s)$

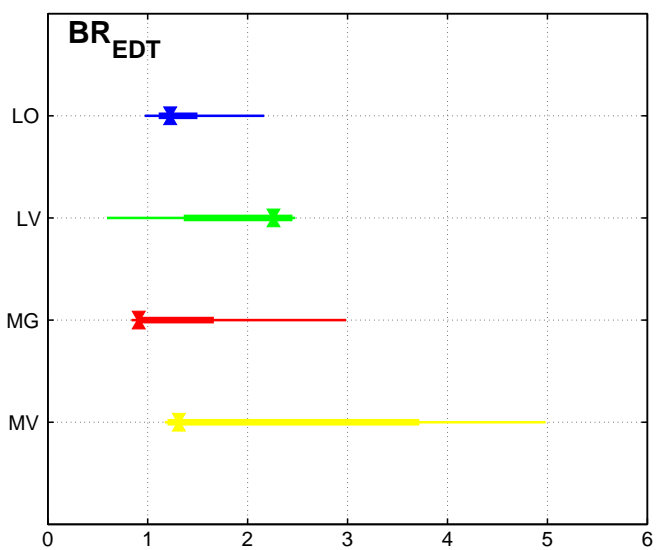

(d) $B R_{E D T}(-)$

Figure 6: $C_{80}, E D T, T_{30}$ and $B R_{E D T}$ at the four different squares plotted in a boxplot in relation to the spatial variability of the parameter. The central mark is the median, the edges of the box are the $25^{t h}$ and $75^{t h}$ percentiles and the whiskers extend to the most extreme data points. LO stands for Oude Markt (Leuven), LV for Vismarkt (Leuven), MG for Grote Markt (Mechelen) and MV for Vismarkt (Mechelen).

smaller. Especially the quantities measured at LO and LV are quite constant over the square. At both of these squares delay-lines are used, which can 
ensure less spatial fluctuation of the parameters, even at a very elongated square such as LO. In Mechelen, no delay-lines were used, causing larger fluctuations in the measured quantities. However, at higher frequencies the deviations from the median value become smaller at MV, the smallest square. This is not the case at MG, the largest square, where obstacles like screens shield measurement locations from high frequencies (MPL5). Since this is not compensated by the use of delay-lines, large variations in the observed parameters were found. The highest values for EDT are found at this square, not only at places shielded from direct sound, but also further away from the speakers.

From these data it can be seen that the smallest squares, MV and LV, have the best behavior for electronically controlled music reproduction. $C_{80}$ is high and $E D T$ low, relative to the other squares. Early reflections contain more energy, since they suffer from less path loss at a smaller square. The use of delay-lines at the large LO helps to ameliorate the parameters at places with inferior acoustics. By this, a less fluctuating parameter course is obtained, in contrast to the largest square at Mechelen.

\subsubsection{Description by $T_{30}$ and $B R_{E D T}$}

As described earlier, $T_{30}$ is independent of measurement position and speaker placement. It is thus an ideal measure to make a comparison of the squares, solely based on their geometry. Generally it is seen that $T_{30}$ increases with the size of the square (fig. 6(c)). The size of LV and MV is comparable (with MV adjacent to a river) and this is reflected in a similar magnitude of $T_{30}$. At LO $T_{30}$ is higher, because of the length of the square. The highest values for $T_{30}$ are found at MG. This is not only caused by its 
dimensions, but also because this square is adjacent to another open area. The broad range of $B R_{E D T}$, although proven to be uncorrelated with $E D T$, is related to the range of fluctuations of EDT. No strong conclusions can be drawn, but it seems that the variation of bass ratio is smallest at squares with a delay-line. The bass ratio extracted out of $T_{30}$ shows to be concentrated around the median (not shown). Somewhat higher values are observed at both squares in Leuven, but a relation with the shape and size of the square is not easy to find.

\subsubsection{Description by $\left[1-I A C C_{E 3 / L 3}\right]$ and $L E V_{\text {calc }}$}

In Fig. 7, the different binaural parameters are shown. Fig. 7(a) depicts $\left[1-I A C C_{E 3}\right]$, which is related to the apparent source width, together with $\left[1-I A C C_{L 3}\right]$, describing the angular dependency of the listener envelopment. For $\left[1-I A C C_{E 3}\right]$ (or $B Q I$ ) the difference between the squares in Leuven and the squares in Mechelen (no delay-lines) is clear. The $\left[1-I A C C_{E 3}\right]$ is higher at both squares in Leuven because of the broadening effect of the delay-lines. $\left[1-I A C C_{L 3}\right]$, on the other hand, is quite high and constant at all squares considered, because the high diffusivity of the façades causes the reverberant sound to reflect in various directions.

Fig. $7(\mathrm{~b})$ shows that $L E V_{\text {calc }}$ differs much more between squares than [1 $\left.I A C C_{L 3}\right]$. The discriminating factor for $L E V_{\text {calc }}$ is not the angular distribution, which is already quite high at all squares, but the difference in relative sound pressure level and $C_{80}$ (eq. 3). $L E V_{\text {calc }}$ is highest at $M G$, the largest square without delay-lines. This can be explained by the large amount of energy still contained in the reverberant field (low $C_{80}$ subtracted from $\Delta L_{\text {eq,oct }}$ in eq. 3). At the smallest square, $M V, L E V_{\text {calc }}$ is lowest (high $C_{80}$ ). However, 
at both squares with delay-lines, the median value of $L E V_{\text {calc }}$ is more or less equal.

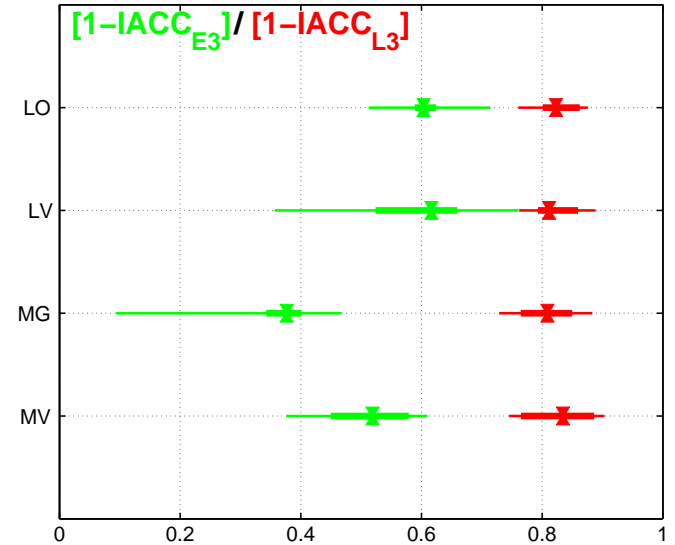

(a) $\left[1-I A C C_{E 3 / L 3}\right](-)$

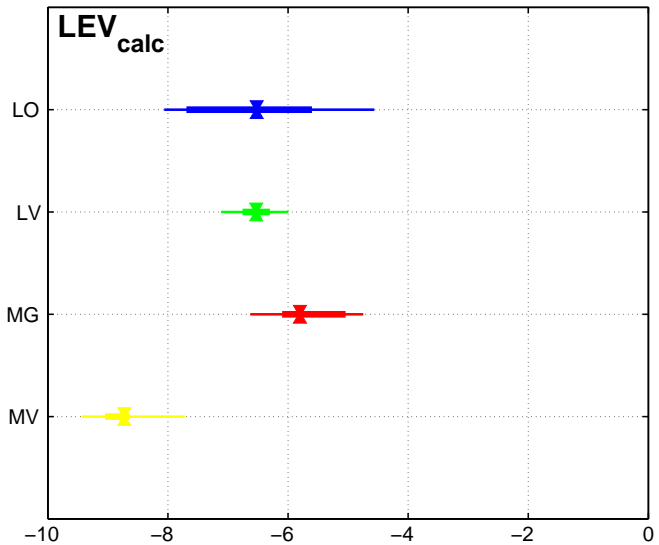

(b) $L E V_{\text {calc }}(-)$

Figure 7: [1-IACC $E 3 / L 3]$ and $L E V_{\text {calc }}$ at the four different squares expressed by boxplots in relation to the variation of the parameter over the square. The central mark is the median, the edges of the box are the $25^{t h}$ and $75^{t h}$ percentiles and the whiskers extend to the most extreme data points. LO stands for Oude Markt (Leuven), LV for Vismarkt (Leuven), MG for Grote Markt (Mechelen) and MV for Vismarkt (Mechelen).

\subsubsection{Description by $\Delta L_{e q, A / C}$}

The last parameters to describe the sound field at the different squares are based on the relative sound pressure level. In contrast to previously discussed parameters, these measurements are performed during the concert and thus include the influence of the audience. In fig. 8 the difference in $L_{e q}$ between different positions at the square and the reference position is plotted for both $A$ - and $C$-weighting for a real life rock music spectrum. At LO, LV and MG measurements were done during two ten-minute periods, of which only the music samples were selected for calculation of $L_{e q}$, and the 
line indicates the average taken over these two measurements. The position of the delay-lines is indicated by the vertical lines.
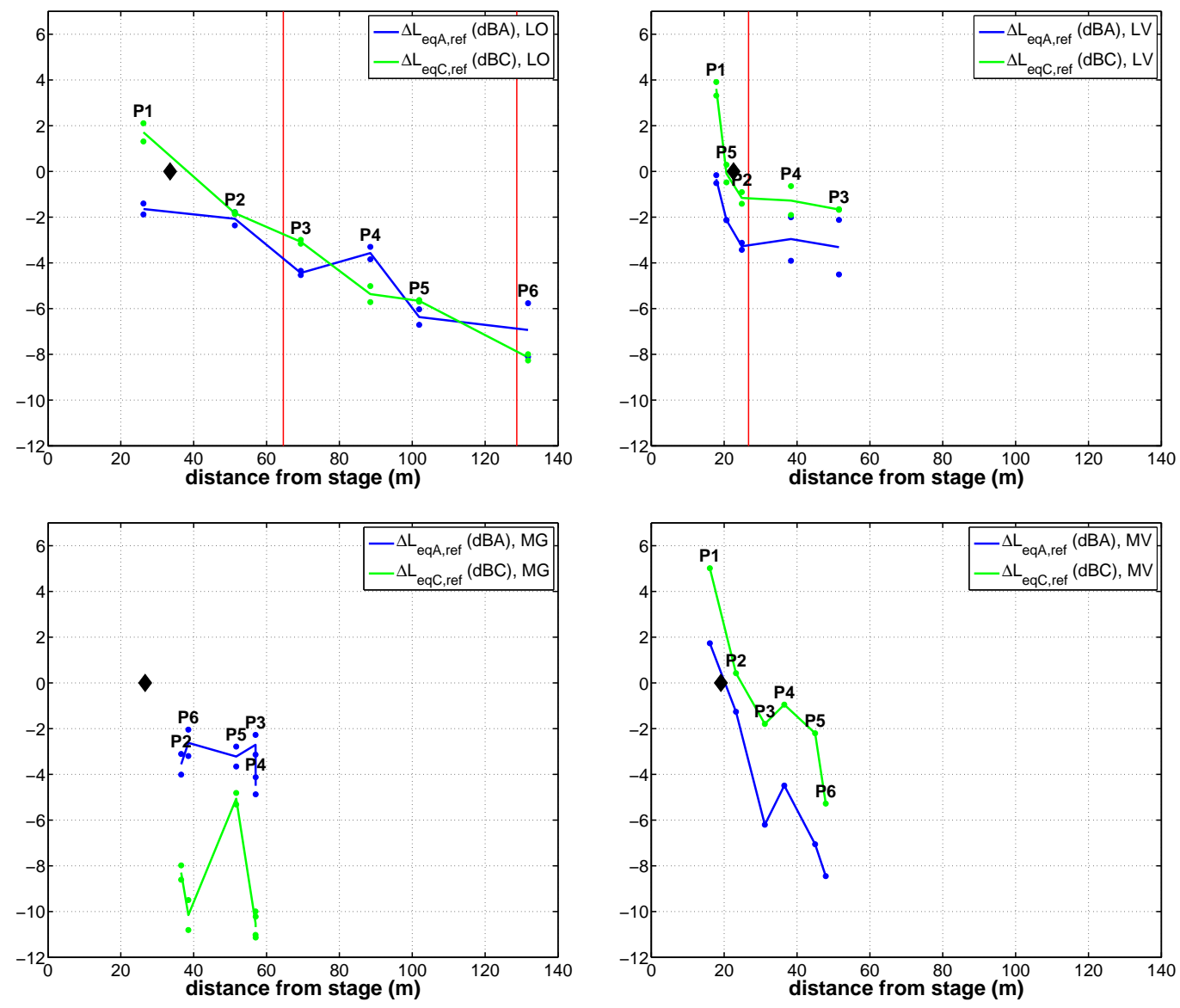

Figure 8: Relative sound pressure level $\left(\Delta L_{e q, A}\right.$ (blue) and $\Delta L_{e q, C}$ (green)) in function of the distance from the stage. The measurement positions are indicated as P1-6 and the reference position is represented by a black diamond-shaped marker. The vertical lines indicate the positions of the delay-lines. LO stands for Oude Markt (Leuven), LV for Vismarkt (Leuven), MG for Grote Markt (Mechelen) and MV for Vismarkt (Mechelen).

As is clear from fig. 8, delay-lines result in a more uniform distribution of 
the sound pressure level over the square. The positive effect of the delay-line at LV is very pronounced. At LO, a linear decrease of level with distance is observed.

At the squares in Mechelen, delay-lines are not present. Although MV is a small square, $\Delta L_{e q}$ decreases rapidly with distance; the half-openness of the square is accounting for this. The distribution of the sound pressure level at MG however jumps out. The fluctuations in $\Delta L_{e q, A}$ are caused by the non-symmetrical square geometry. Since the back of the square is only halfway closed, as it borders another square, more energy is lost at this side, compared to the energy loss at the closed side. This accounts for low levels at MPL2 and MPL4, in contrast to MPL3 and MPL6, which are located close to reflective structures.

From the correlation analysis, it was concluded that the correlation between $\Delta L_{e q, A}$ and $\Delta L_{e q, C}$ is small. This is reflected in differences in distribution of both parameters over the squares. For $L O, L V$ and $M V$ the course of $\Delta L_{e q, A}$ and $\Delta L_{e q, C}$ with distance from the stage contains limited, but noticeable, differences. At MG however, high differences are observed when applying different frequency weighting schemes. In A-weighted levels, acoustical energy in the lower octave bands is less important. When looking at $\Delta L_{e q, C}$ in function of the distance to the stage, distinct peaks are found at MPL2 and MPL5, in contrast to the A-weighted level. These findings were confirmed during a second sound pressure level measurement campaign at MG in 2010 (not shown). An explanation can be found in the specific situation at this square. Points MPL1/REF, MPL2 and MPL5 are situated above an underground parking lot, which could act as a kind of coupled res- 
onator. The other points are less sensitive to these vibrations since these are at the border of the parking lot. Indeed, many bystanders were complaining about annoying low frequency vibrations. These vibrations could even be felt on the square surface during music passages with a large amount of low frequency energy.

Based on these findings, it is clear that frequency weighting should not be restricted to A-weighting. The use of C-weighted sound pressure levels gives added value to the evaluation of the sound field, given the often prominent low frequencies at rock concerts.

\section{Conclusions}

In this study, an extensive measurement campaign has been set up to characterize the sound field at urban squares. Different acoustical parameters were measured before and during rock concerts held at such squares. From this data different statistically independent factors were identified by correlation analysis. It was found that $C_{80}, T_{s}$ and EDT are highly correlated, but not correlated with $T_{30}$, in contrast to findings in performance halls $[12,13]$. At rock concerts held on urban squares, $\Delta L_{e q, A}$ and $\Delta L_{e q, C}$ were also found to have a low correlation. The investigated independent parameter set contained $C_{80}, T_{30}, B R,\left[1-I A C C_{E 3 / L 3}\right], L E V_{\text {calc }}$ and both $\Delta L_{e q, A}$ and $\Delta L_{e q, C}$. This selection of parameters is obviously influenced by the set that was initially selected. Other researchers [8,9] started from different sets and found comparable dimensionality. In particular $T_{\text {sub }}, I A C C$ and listening level are closely related to $T_{30}, I A C C_{E / L}$ and relative sound level respectively. 
In order to estimate the influence of the delay-lines on the sound field, measurements were done at an open festival site. It is clearly seen that the effect on the parameters, except for $T_{30}$, strongly depends on the considered measurement positions. Near the line-arrays, an improvement is seen. However, at distant locations, the quality decreases due to large time-shifts between the arrival of sound coming from different sources. This became clear when evaluating $C_{80}$. As for $\left[1-I A C C_{E 3}\right]$, an increase is seen at all locations when delay-lines are introduced.

$T_{30}$ is found to be an interesting parameter to compare the sound field at different squares. This parameter is strongly dependent on the square geometry. Furthermore, it was shown that $T_{30}$ is only little dependent on the location at the square and is almost not influenced by the amplification system and the presence of delay-lines.

A comparison between four urban squares is made, based on statistically independent acoustical parameters extracted from impulse response measurements (before the concerts) and sound pressure level measurements (during the concerts). In absence of distributed speaker arrays, the sound quality at smaller squares is higher than at larger squares. The introduction of delaylines can greatly improve the acoustics, even at large elongated squares. For the design of these speaker systems, the sound engineer can make use of different parameters. $C_{80}$ tends to be most dependent on the considered location and speaker set-up. Squares with delay-lines also have higher [1-IACC $E 3]$, related to $A S W$ and $B Q I$. However, concerning $L E V_{\text {calc }}$, the determining factor is $G_{L}\left(\Delta L_{e q, o c t}\right)$ and not the angular distribution, which is already high at all squares. Relative sound pressure level measurements are useful 
to describe the level decrease over the square. The use of C-weighted levels was shown to indicate potential problems with strong low frequency passages during concerts. The distribution of the acoustical energy over time cannot be derived when considering relative sound pressure levels only. Nevertheless, this parameter is nowadays the only commonly used design parameter. In this paper, room acoustical parameters were measured at urban squares in order to characterize them acoustically. Statistically independent parameters that are sensitive to the specific square geometry and amplification system were identified. Further research should link these parameters to a subjective evaluation of the sound quality at different squares and give more insight in the preferred parameter values for rock music [34,35]. Related studies have already been undertaken for rock concert halls $[3,36]$ and classical concert halls $[8,11]$, but not yet for open air concerts at public squares.

[1] D. Paini, A. Gade, J. Rindel, Is reverberation time adequate for testing the acoustical quality of unroofed auditoriums?, Proc. of IoA 28 (2006) $66-73$.

[2] L. beranek, Audience and seat absorption in large halls, J. Acoust. Soc. Am. 32 (1960) 661-670.

[3] N. Adelman-Larsen, E. Thompson, A. Gade, Acoustics in rock and pop music halls, AES 112th Convention.

[4] L'Acoustics - soundvision, http://www.1-acoustics.com/.

[5] Meyer - MAPP Online Pro, http://www.meyersound.com/. 
[6] M. Schroeder, D. Gottlob, K. Siebrasse, Comparative study of European concert halls: correlation of subjective preference with geometric and acoustic parameters, J. Acoust. Soc. Am. 56 (1974) 1195-1201.

[7] L. Cremer, H. Mueller, Principles and applications of room acoustics, Hirzel, Stuttgart, 1978.

[8] Y. Ando, Calculation of subjective preference at each seat in a concert hall, J. Acoust. Soc. Am. 74 (1983) 873-887.

[9] M. Tohyama, H. Suzuki, Y. Ando, The nature and technology of acoustic space, Academic Press, 1995.

[10] L. Beranek, Concert halls and opera houses, Springer New york, 2004.

[11] L. Beranek, Subjective rank-orderings and acoustical measurements for fifty-eight concert halls, Acta Acustica united with Acustica 89 (2003) $494-508$.

[12] A. Gade, Prediction of room acoustical parameters, J. Acoust. Soc. Am. 89 (1991) 1857(A).

[13] S. Cerda, A. Gimenez, J. Romero, R. Cibrian, J. Miralles, Room acoustical parameters: A factor analysis, Applied Acoustics 70 (2009) 97-109.

[14] Acoustics - Measurement of room acoustic parameters - Part 1: Performance spaces, ISO 3382-1:2009(E) (2009).

[15] Y. Ando, D. Gottlob, Effects of early multiple reflections on subjective preference judgments of music sound fields, J. Acoust. Soc. Am. 65 (1979) 524-527. 
[16] Acoustics - Application of new measurement methode in building and room acoustics, ISO 18233:2006(E) (2006).

[17] T. Hidaka, L. Beranek, T. Okano, Interaural cross-correlation, lateral fraction and low- and high-frequency sound levels as measures of acoustical quality in concert halls, J. Acoust. Soc. Am. 98 (1995) 988-1007.

[18] M. Barron, The subjective effects of first reflections in concert halls The need for lateral reflections, J. Sound. Vib. 15 (1971) 475-494.

[19] M. Barron, A. Marshall, Spatial impression due to early lateral reflections in concert halls, J. Sound. Vib. 77 (1981) 211-232.

[20] J. S. Bradley, G. A. Soulodre, Objective measures of listener envelopment, J. Acoust. Soc. Am. 98 (1995) 2590-2597.

[21] G. Soulodre, M. Lavoie, S. Norcross, Objective measures of listener envelopment in multichannel surround systems, J. Audio Eng. Soc. 51 (2003) 826-840.

[22] J. Ryu, J. Jeon, Subjective and objective evaluations of a scattered sound field in a scale model opera house, J. Acoust. Soc. Am. 124 (2008) $1538-1549$.

[23] L. Beranek, Concert hall acoustics - 2008, J. Audio Eng. Soc. 56 (2008) $532-544$.

[24] M. Schroeder, New method of measuring reverberation time, J. Acoust. Soc. Am. 37 (1965) 409-412. 
[25] T. Hidaka, T. Okano, L. Beranek, Interaural cross correlation (IACC) as a measure of spaciousness and envelopment in concert halls (a), J. Acoust. Soc. Am. 92 (1992) 2469.

[26] T. Okano, L. Beranek, T. Hidaka, Relations among interaural crosscorrelation coefficient, lateral fraction, and apparent source width in concert halls, J. Acoust. Soc. Am. 104 (1998) 255-265.

[27] M. Morimoto, Z. Maekawa, Effects of low frequency components on auditory spaciousness, Acustica 66 (1988) 190-196.

[28] L. Beranek, Concert and opera halls, Acoust. Soc. Am., 1996.

[29] G. Stan, J. Embrechts, D. Archambeau, Comparison of different impulse response measurement techniques, J. Audio Eng. Soc. 50 (2002) 249-262.

[30] Specification for octave-band and fractional-octave-band analog and digital filters, ANSI S1.11-2004 (2009).

[31] A. Lundeby, T. Vigran, H. Bietz, M. Vorländer, Uncertainties of measurements in room acoustics, Acustica 81 (1995) 344-355.

[32] W. T. Chu, Comparison of reverberation measuerements using Schroeder's impulse method and decay-curve averaging method, J. Acoust. Soc. Am. 63 (1978) 1444-1450.

[33] D. Paini, J. Rindel, A. Gade, G. Turchini, The acoustics of public squares/places: a comparison between results from a computer simulation program and measurements in situ, internoise 2004. 
[34] Y. Ando, T. Okano, Y. Takezoe, The running autocorrelation function of different music signals relating to preferred temporal parameters of sound fields, J. Acoust. Soc. Am. 86 (1989) 644-649.

[35] S. Kaieda, K. Kawai, T. Yano, Y. Ando, A study on measures of timbre of electric guitar sounds in terms of power spectrum and auto correlation function, J. Temporal Des. Arch. Environ. 9 (2009) 43-46.

[36] N. Adelman-Larsen, E. Olmos, A. Gade, Acoustics in halls for rock music, Joint Baltic-Nordic Acoustics Meeting 2004. 